\section{Ischemic heart disease in the emergency room: state of the art, innovation and research}

\author{
Giuseppe Lippi, ${ }^{1}$ Mario Cavazza, ${ }^{2}$ \\ Andrea Peracino, ${ }^{3}$ Marco Tubaro, ${ }^{4}$ \\ Alexandru Nechita, ${ }^{5}$ Paola Ballarino, ${ }^{6}$ \\ Frank W. Peacock, ${ }^{7}$ Salvatore Di Somma 8,9 \\ 'Laboratory of Clinical Chemistry and \\ Hematology, Department of Pathology and \\ Laboratory Medicine, Parma University \\ Hospital; ' ${ }^{2}$ Emergency Department, S. \\ Orsola-Malpighi University Hospital, \\ Bologna, Italy; ${ }^{3}$ Giovanni Lorenzini Medical \\ Science Foundation, Milano, Italy, and \\ Houston, TX, USA; ${ }^{4}$ Intensive Cardiac Care \\ Unit, Cardiovascular Department, San \\ Filippo Neri Hospital, Roma, Italy; \\ ${ }^{5}$ Cardiology Department, Sf. Pantelimon \\ Emergency Clinical Hospital, Bucharest, \\ Romania; ' ${ }^{6}$ Emergency Department, San \\ Martino University Hospital, Genova, Italy; \\ ${ }^{7}$ Emergency Medicine Section, Baylor \\ College of Medicine, Houston, TX, USA; \\ ${ }^{8}$ Emergency Department, Sant'Andrea \\ Hospital; ${ }^{9}$ Great Network - Global Research \\ on Acute Conditions Team, Roma, Italy
}

\section{Abstract}

This opinion paper is aimed to provide an overview about the state of the art, innovation and research in ischemic heart disease in the emergency room, and is a synopsis of the lectures of the $3^{\text {rd }}$ Italian GREAT Network Congress (Rome, 15-19 0ctober 2012). The leading issues of a multidisciplinary risk stratification and diagnosis of patients presenting to the emergency department with suspected ischemic heart disease will be discussed taking into consideration the variable onset of clinical signs and symptoms, the role of novel highly-sensitive troponin immunoassays, the promising use of an 80-lead electrocardiogram, echocardiography and risk stratification scores. Preliminary information will also be provided about the ongoing Italian multicentric registry on chest pain patients in emergency department, an observational prospective study aimed to collect data about patients presenting at the emergency department with typical chest pain suggesting an acute coronary syndrome.

\section{Introduction}

According to the most recent statistics of the
American Heart Association (AHA), the overall prevalence of ischemic heart disease (IHD) in the US is $7 \% .{ }^{1}$ It is also estimated that nearly 800,000 US inhabitants will experiment a new coronary event, whereas additional 195,000 silent first acute myocardial infarctions (AMIs) may occur each year. As such, an US inhabitant is expected to have a coronary event every 25 sec, and someone will die for this nearly every minute. $^{1}$

Hence, it is no surprise that patients with sudden onset of chest pain and suspected acute myocardial infarction (AMI) represent one of the largest subset urgently admitted to the emergency department (ED). In Italy, around 1 million patients each year present to the ED with chest pain symptoms. Nearly half of these $(\sim 45 \%)$ have an acute coronary syndrome (ACS), whereas $\sim 18 \%$ are finally diagnosed with AMI. ${ }^{2}$

One of the biggest clinical challenges in IHD is the timely distinguishing of patients who may benefit most from urgent management from those who can be safely excluded for extensive diagnostic testing and invasive procedures. There have been remarkable advances in understanding the pathophysiology of AMI over the past decades, and this has led to the development of innovative diagnostic and imaging techniques, the establishment of specific chest pain units, guided protocols and risk scores aimed at helping the clinician to better stratify patients with suspected AMI. In these emerging scenarios, biomarkers have played an essential role for diagnosis and prognostication, being also key components of the most recent definition of AMI, even if chest pain management remains an unresolved dispute for different clinical specialists such as emergency physicians, cardiologists, laboratory professionals, radiologists, and healthcare administrators. ${ }^{3}$ The foremost need in ED is to quickly and accurately rule-out AMI in acute setting. The clinical and therapeutic decision making is often done in critical conditions, namely limited resources, patient overcrowding, frantic and confusing environments. Additional challenges are represented by a growing trend towards ED admission of aged and complicated patients, who often carry several co-morbidities which mimic an ACS, or whom may have an atypical presentation of myocardial ischemia characterized by non specific signs and symptoms. With most countries still experiencing recession due to the current huge economical crisis, the substantial costs for diagnosis and management of patients with IHD pose other important hurdles to the effective management of chest pain in ED.

Additional challenges will soon emerge, after a broad implementation of the new European guidelines. ${ }^{4,5}$ Thus, it will become essential to reorganize and harmonize the clinical pathways throughout the various national healthcare systems, along with guarantee of complain by dif-
Correspondence: Salvatore Di Somma, Emergency Department, Sant'Andrea Hospital, via di Grottarossa 1035-1039, 001891 Roma, Italy. Tel. +39.06.3377.5581 - Fax: +39.06.3377.5890.

E-mail: salvatore.disomma@uniromal.it

Key words: ischemic heart disease, myocardial infarction, biomarkers, electrocardiography, risk stratification.

Contributions: the authors contributed equally.

Conflict of interests: the authors declare no potential conflict of interests.

Received for publication: 15 February 2013.

Accepted for publication: 8 March 2013.

This work is licensed under a Creative Commons Attribution 3.0 License (by-nc 3.0).

(C) Copyright G. Lippi et al., 2013

Licensee PAGEPress, Italy

Emergency Care Journal 2013; 9:e7

doi:10.4081/ecj.2013.e7

ferent healthcare organizations. It will also become necessary to establish whether (and eventually to what extent) the new guidelines will be effective to reduce the door-to-balloon time ${ }^{6}$ which is supposed to be the contribution of new imaging techniques and novel biomarkers in different settings and specific realities, and whether the continuity of care will assume a better organized role in consolidating in-hospitals care. It is also noteworthy that additional challenges will emerge from the integration of the EDs within novel re-organized networks of coronary units, namely according to the hub and spoke paradigm. Finally, it will also be important to verify whether the implementation of new guidelines will meet the different scientific clinical, ethical and legal expectations raised by the various stakeholders. We do hope that answers to these questions may be found in the pages of this document. This collective, opinion paper is thereby aimed to provide an overview about the state of the art, innovation and research on ischemic heart disease in the emergency room, and it is a synopsis of the lectures of $3^{\text {rd }}$ Italian GREAT Network Congress (Rome, 15-19 October 2012).

\section{Case Report}

\section{Optimization of rule-out} of acute coronary syndrome in the emergency room

Patients with chest pain admitted to the ED have a variety of diseases, and only few of them have an underlining ACS. As such, the first task of the ED physician is to make a diagnosis to 
distinguish between IHD and other serious diseases, in order to rule out myocardial ischemia and thereby discharge early and safely those patients who do not require hospital care. When the rule-out process produces a negative result, the rule-in is more complicated, and should take into account both the diagnosis and the prognostic stratification, in order to identify the appropriate ward that the patient should be admitted to and delineate a meaningful diagnostic and therapeutic pathway.

The first step in the diagnostic approach of patients with chest pain admitted to the ED encompasses an accurate history collection and careful physical examination, including an estimate of the patient probability to have an ACS, pain characteristics and the presence of specific ACS risk factors. ${ }^{7}$ An electrocardiogram (ECG) should be recorded as soon as possible from patient admission, and interpreted by an experienced healthcare professional. In case of persistent ST-segment elevation accompanied by signs and symptoms of IHD [i.e. ST-segment elevation myocardial infarction (STEMI)], no additional tests are necessary to complement the diagnosis and the patient should be immediately treated with reperfusion strategy [primary percutanous coronary intervention (PCI), or thrombolysis as a second choice].$^{8}$ In the presence of ST depression and/or T wave inversion, the probability of ACS is high and the measurement of cardiac biomarkers is useful for the differential diagnosis between unstable angina and non-ST-elevation myocardial infarction (NSTEMI), as well as for prognostic stratification.

According to the most recent guidelines of the European Society of Cardiology (ESC), American College of Cardiology Foundation (ACCF), AHA and World Heart Federation (WHF), ${ }^{4,5,9}$ the diagnostic approach of patients with suspected IHD is principally based on the assessment of cardiospecific troponins (either T or I), preferably using a sensitive or highlysensitive (HS) immunoassay. A paradigm shift has occurred with the introduction of the novel HS immunoassays, whose remarkably improved analytical sensitivity has enabled to reduce the limit of detection of previous contemporary sensitive immunoassays by 5 to over 10 times,,$^{10}$ so that physiological troponin values may now be measurable in more than $80 \%$ of presumably healthy subjects (compared to 1 to $30 \%$ with contemporary sensitive methods). ${ }^{11}$ On the one hand, the improved sensitivity and negative predictive value enable an early and accurate rule-out of AMI in the ED when test results are negative, typically sooner $(6 \mathrm{~h})$ than conventional methods. ${ }^{12}$ On the other hand, the ability to measure troponin values in the vast majority of patients admitted to the ED - some of whom displaying values above the $99^{\text {th }}$ percentile of the upper reference limit (URL) of a normal population - decreases the specificity and positive predictive value for myocardial ischemia because an increased troponin concentration may be sustained by a variety of other (typically cardiac) conditions. ${ }^{13}$ Accordingly, a more accurate interpretation of signs, symptoms and ECG findings is necessary for preventing over-diagnosis or misdiagnosis of myocardial infarction (MI), especially NSTEMI. Another essential aspect is the use of the so-called delta biomarker approach, according to which troponin values must not be considered a static measure, but rather they should be used in a kinetic pattern where a progressively increasing concentration is suggestive for an evolving myocardial damage (typically an AMI), whereas mostly unchanged values are indicative for stable cardiac disease. The still unresolved problems here are: i) the identification of an appropriate variation threshold, which should be inherently dependent upon several biological variables such as age, gender and ethnicity; and ii) the possible use of absolute (i.e. percentage) variation. ${ }^{14,15}$

When a high suspicion for ACS remains after non-diagnostic HS troponin results and ECG findings, stress-imaging testing (i.e. stress echo or myocardial scintigraphy) can also be used in the ED, while for differential diagnosis of structural cardiac or vascular diseases, a multidetector computer tomography (CT) scan or a cardiac magnetic resonance can help to confirm and/or rule out a diagnosis of pulmonary embolisms, aortic dissection, myocarditis and other conditions. Although the rule-out of AMI should mostly rely on non-diagnostic values of HS troponin immunoassays (i.e. lower than $99^{\text {th }}$ percentile URL) after 3 to $6 \mathrm{~h}$, the exclusion of ACS (including unstable angina) needs a multifaceted approach, entailing clinical judgment on history, physical examination, and ECG. ${ }^{16}$ It is also noteworthy that ruling out an ACS does not necessarily entails that the patient does not suffer from an another serious disease. In this respect, the meaningful clinical judgment has a pivotal role for best managing patients with chest pain in the ED.

\section{Biomarkers for early diagnosis of acute coronary syndrome: only troponin?}

According to the working group of the National Institute of Health (NIH), a biological marker is defined as a characteristic objectively measured and assessed as an index of a physiological or pathological process, as well as an indicator of pharmacologic response to therapy. ${ }^{17}$ Biomarkers can hence be assessed for a vast array of purposes, including screening and diagnosis of patients with certain pathologies or abnormal conditions, classification, staging and prognostication of diseases and prediction or monitoring of therapeutic responses. According to recent and reliable evidence, patients presenting to the ED with suspected ACS can receive a final diagnosis of AMI in less than $3 \mathrm{~h}$, with both diagnostic sensitivity and a negative predictive value of $100 \%$. To do this, methods characterized by an appropriate analytical sensitivity capable of obtaining measurable values in at least $50 \%$ of subjects are used. ${ }^{18}$ It is noteworthy, however, that neither the sensitivity nor the negative predictive value of HS immunoassays achieve a value of $100 \%$ in samples collected on ED admission, so that the search for diagnostic algorithms combining troponin(s) with early biomarkers may still be seen as an appealing perspective. $^{19}$

A variety of tests have been proposed over the past decades for the diagnostic approach of IHD, either alone or in combination with troponin..$^{20}$ These mostly - but not exclusively - include inflammatory mediators or reactants (i.e. highsensitive $\mathrm{C}$ reactive protein, procalcitonin, cytokines and interleukins), indices of plaque instability (i.e. platelet-leukocyte aggregates, CD40 ligand), biomarkers of ischemia (i.e. ischemia-modified albumin, heart-fatty acidbinding protein, pregnancy-associated plasma protein-A, choline, free fatty acids), markers of myocardyocite necrosis (i.e. myoglobin, creatine kinase isoenzyme MB, glycogen phosphorylase BB, circulating microRNAs), cardiac hormones or peptides (i.e. adrenomedullin and mid-regional pro-adrenomedullin, copeptin, neopterin, natriuretic peptides), thrombotic biomarkers (i.e. D-dimer, fibrin/fibrinogen degradation products, thrombus precursor protein, soluble fibrin monomer, prothrombin fragment $1+2$, thrombin-antithrombin and plasminantiplasmin complexes), as well as other less specific indices such as mean platelet volume (MPV), red blood cell distribution width (RDW) or plasma glucose. ${ }^{21,22}$

Although a huge amount of data has been produced and published on the clinical usefulness of these parameters, it seems reasonable to conclude that the growing development and clinical use of HS troponin immunoassays will dramatically limit the practical significance of the measurement of additional biomarkers for risk stratification and early diagnosis of IHD. This is mainly attributable to the fact that the diagnostic accuracy of most of these tests is comparable - at best - to that of HS troponin immunoassay. Indeed, some of them require research technologies that are currently unsuitable for large volumes and optimal turnaround time (e.g. manual immunoassays, cumbersome separative techniques, flow cytometry), others are simply too expensive to comply with the limitations of government funding that will be a hallmark of national healthcare systems for several years to come. Medium- and long-term risk stratification of both cardiac and non-cardiac 
complications offer a more reasonable and pervasive perspective for biomarkers of cardiac functions. Among them, natriuretic peptides, mid-regional adrenomedullin and copetin, for example, may help identify those patients with an increased risk of heart and renal impairment after an AMI, thus enabling a tailored treatment and a better outcome. ${ }^{23,24}$

\section{Multi-leads electrocardiogram evaluation of acute chest pain in the emergency department}

As previously mentioned, the evaluation of patients with symptoms of suspected ACS who present to the ED is expensive and time consuming, and always starts with the 12-lead ECG. This rapid, non-invasive and relatively inexpensive diagnostic tool should be performed at the earliest, preferably within 10 min from patient presentation. When its findings are diagnostic for an ACS, a series of recommended clinical interventions should follow. However, with existing technology, the 12lead ECG is non-diagnostic in approximately $50 \%$ of patients. ${ }^{25}$ Thereby, the currently available ECG cannot be considered a precise tool. Even when findings of an AMI are detected, only $90 \%$ of patients will be found to have catheterization and biomarker findings consistent with this diagnosis. ${ }^{26}$ Conversely, when used as a screening tool, the 12-lead ECG has a sensitivity for AMI in the range 25 to $57 \%$.

Though practically unchanged since 1940 , great improvements in computer processing technology have transformed the 12-lead ECG into a device capable of performing electrical body surface mapping. This is accomplished by the application of 80 ECG leads placed circumferentially on the patient thorax. The data obtained can be presented as 80 individual lead tracings, or alternatively color-coded, and projected onto the image of a thorax. Following a scheme where green represents normal baseline ST segments, red represents ST elevation, and blue ST depression, the presence of AMI can be rapidly identified. When body surface mapping information is presented in this latter fashion, the use of 80-lead ECG provides a remarkable $81 \%$ increase in sensitivity for detecting AMI compared to a standard 12-lead ECG. ${ }^{27}$ Most improvements in sensitivity with the 80-lead ECG occur as a result of superior detection of AMI in those anatomic regions known to be challenging for the 12-lead ECG. For example, this includes a 13.5 fold improvement in the rate of a correct diagnosis of posterior AMI, and a 1.4 times improvement in detecting right ventricular (RV) infarcts, even when a V4R lead is added to the routine 12lead ECG.

When considering the entire AMI population, only $33 \%$ is found to suffer STEMI with conventional ECG. However, by using the 80 - lead ECG, the rate of detected STEMI increases to $50 \%$. An improved detection of patients suffering from acute STEMI is able to identify those in whom early intervention designed to establish reperfusion [either percutaneous coronary intervention (PCI) or thrombolysis] may provide additional clinical benefit. It is noteworthy that because 80-lead ECG is mechanically performed in identical fashion as the 12-lead, similar challenges exist in obtaining clear tracing in excessively hirsute, diaphoretic, or morbidly obese patients. When this occurs, the usual strategies for sticker adherence must be employed. Similarly to the 12-lead ECG, the 80-lead ECG can suffer from interpretation confounders. This may need the clinical correlation to differentiate suspected acute ST segment changes consistent with AMI in the setting of pericarditis, benign early repolarization, left ventricular (LV) aneurysm, left bundle branch block (LBBB) and others, although the increased number of leads may provide insight as to the correct diagnosis that is not available with the 12-lead.

In ED, tools to allow early safe discharge of suspected ACS patients would be imminently useful, and thus the utility of this application of body surface mapping has been examined. In a secondary analysis of the OCCULT trial, ${ }^{28}$ 1816 patients prospectively defined as being at low to moderate risk of ACS were risk stratified by initial laboratory findings and an 80-lead ECG. Patients were excluded if they had an initially elevated troponin $(\mathrm{n}=217)$, creatinine $>2.0 \mathrm{ng} / \mathrm{mL}(\mathrm{n}=52)$, or if the 80-lead ECG was abnormal $(n=156)$. Thus, from the original group, $87.9 \%$ patients remained and they could be categorized shortly after arrival as presumptively low risk. When the 30 day outcomes of this low risk patient group were evaluated, there were $0.4 \%$ deaths, $0.8 \%$ AMI, and $2.4 \%$ re-hospitalized. ${ }^{28}$ This suggests that, combined with initial laboratory findings, the 80-lead ECG may identify a population of early ED discharge candidates.

\section{Discussion}

Diagnosis and risk stratification of IHD is challenging, and may often result in a very high number of inappropriate admissions. On the other side, $2 \%-10 \%$ of the patients discharged as non coronary chest pain experience an AMI, with a mortality rate that is 2-fold higher than that of patients admitted to the hospital. ${ }^{1}$ According to these concerning figures, the evaluation of patients in ED should always be made within the rules of AMI networks established in each country or region. Though STEMI networks are very clearly defined, less defined protocols exist for NSTEMI and unstable angina. In some countries, all patients with ECG changes and/or positive troponin results are referred to the catheterization laboratory within the next $24 \mathrm{~h}$.

The diagnostic process is multifaceted and take profit from a variety of complementary tools, such as clinical examination, ECG, biomarkers, echocardiography, other imaging and stress tests. Although the role of biological markers and ECG has already been clearly emphasized so far, echocardiography has the peculiar potentiality to detect $\mathrm{LV}$ wall motion disorders in patients with non-diagnostic ECG and normal troponin values, and this is the only abnormality that can be found in some patients. Echocardiography can detect global LV dysfunction, early complications of AMI, and may also provide a fast answer about other etiologies of chest pain such as aortic dissection, pericarditis or pulmonary embolism. ${ }^{29}$ The use of risk stratification scores such as the thrombolysis in myocardial infarction (TIMI) risk score or the global registry of acute coronary events (GRACE) score, can both be indicative or misleading, depending on the patient's characteristics. $^{30}$ It is unquestionable, however, that a patient with a high TIMI score has a high probability of an adverse outcome, whereas the GRACE score may provide additional hemodynamic information. Basically, the GRACE score provides information on the risk of in-hospital death, 6-month-death and also helps to address the patient with NSTEMI towards immediate or delayed coronary intervention. The GRACE score was validated on a big cohort of patients, showing a very big prognostic difference for those patients with a score $>140$ points, especially when treated by an early invasive strategy. ${ }^{31}$

Patients presenting with ACS complicated by acute heart failure are a very high risk population, which may receive the highest benefits from acute revascularization. In daily practice, this strategy is difficult to be established, and this is due to objective difficulties in identifying a personalized therapeutic management for these patients. All the diagnostic tools should hence be used in patients with suspected ACS, since a tailored diagnostic workup remarkably increases the diagnostic accuracy, the appropriateness and efficacy of treatment, and it finally improves the outcomes effectively. ${ }^{32}$

\section{Conclusions}

Although some registries already exist about the management and treatment of patients with ACS in cardiology settings, ${ }^{5}$ there is still a lack of published information about patients with chest pain in the ED setting. Moreover, international guidelines for approaching chest pain in patients referred to the ED exist, still they are applied quite differ- 
ently all over the world, or even among different EDs of the same country. This is mainly due to the different organization of EDs, where the presence of cardiologist(s) is granted in some cases, but not in others. To describe the current approach to chest pain in EDs in Italy, the GREAT network has thereby designed an observational registry.

The Italian multicentric registry on chest pain patients in ED is an observational prospective study aimed to collect data about patients presenting to the ED with typical chest pain in different Italian centers. The objective is to enroll more than 10,000 patients in 10 different centers (Genoa, Parma, Rome, Milan, Pavia, Naples, Catania, Bologna, Venice and Novara), and include at least 2000 patients with a final diagnosis of ACS. In this registry, patients data will be collected according to specific areas of interest, i.e. age, gender, haemodynamic parameters (e.g. arterial blood pressure, heart rate, oxygen saturation, sinus rhythm, additional symptoms, killip class, etc.), ECG findings, risk factors (history of coronary artery disease, smoke, hypercholesterolemia, body mass index, hypertension, diabetes, family history, chronic kidney disease), drugs taken at home, results of laboratory workout and, in particular, biomarkers testing at $0,3,6$ and eventually $12 \mathrm{~h}$ after ED admission (other cardiac biomarkers may be included in the schedule after the beginning of the study). Moreover, the patient final disposition, final diagnosis, medications administered in ED, risk scores such as TIMI or GRACE risk score, and re-hospitalization after discharge will be recorded.

Since the quantitative assessment of chest pain is one of the most debated topics in the literature, chest pain classification (classified from 1 to 3 according to the probability to be typical for ACS) will be also evaluated. On the basis of these registry results, it would be possible to compare them with the data from other existing cardiology registries. The peculiarity of this registry would also include the possibility to present a picture of the true population referring to ED for chest pain patients in Italy and the different approach for rule-out and rule-in of the ED physicians. The study is expected to start in January 2013 and concluded within 1 year. Preliminary data will be presented to next GREAT meeting in Rome, October 2013.

\section{References}

1. Roger VL, Go AS, Lloyd-Jones DM, et al. American heart association statistics committee and stroke statistics subcommittee. Executive summary: heart disease and stroke statistics 2012 update: a report from the American heart association. Circulation 2012;125:188-97.

2. Bracco C, Melchio R, Sturlese U, et al. Early stratification of patients with chest pain and suspected acute coronary syndrome in the emergency department. Minerva Med 2010;101:73-80.

3. Lippi G, Cervellin G. [The clinical role of high-sensitive troponin assays: update, interpretation, and perspectives]. [Article in Italian]. Recenti Prog Med 2010;101: 423-8.

4. Steg PG, James SK, Atar D, et al. ESC Guidelines for the management of acute myocardial infarction in patients presenting with ST-segment elevation: the task force on the management of ST-segment elevation acute myocardial infarction of the European Society of Cardiology (ESC). Eur Heart J 2012;33:2569-619.

5. Hamm CW, Bassand JP, Agewall S, et al. ESC Guidelines for the management of acute coronary syndromes in patients presenting without persistent ST-segment elevation: the task force for the management of acute coronary syndromes (ACS) in patients presenting without persistent ST-segment elevation of the European Society of Cardiology (ESC). Eur Heart J 2011;32:2999-3054.

6. Comelli I, Vignali L, Rolli A, et al. Achievement of a median door-to-balloon time of less than 90 minutes by implementation of organizational changes in the 'Emergency Department to Cath Lab' pathway: a 5-year analysis. J Eval Clin Pract 2012;18:788-92.

7. Fox KAA, McLean S. NICE guidance on the investigation of chest pain. Heart 2010;96: 903-6.

8. Lippi G, Franchini M, Cervellin G. Diagnosis and management of ischemic heart disease. Semin Thromb Hemost (2013) (in press).

9. Thygesen K, Alpert JS, Jaffe AS, et al. Writing group on behalf of the joint ESC/ACCF/AHA/WHF task force for the universal definition of myocardial infarction. Third universal definition of myocardial infarction. J Am Coll Cardiol 2012;60:1581-98.

10. Lippi G, Cervellin G. Standardized designation of commercial troponin immunoassays. Kardiol Pol 2012;70:1095-6.

11. Apple FS, Ler R, Murakami MM. Determination of 19 cardiac troponin I and T assay 99th percentile values from a common presumably healthy population. Clin Chem 2012;58:1574-81.

12. Reichlin T, Hochholzer W, Bassetti S, et al. Early diagnosis of myocardial infarction with sensitive cardiac troponin assays. New Engl J Med 2009;361:858-67.

13. Lippi G, Cervellin G. Identification of tro- ponin determinants for improving its diagnostic performance in the Emergency Department. J Emerg Med 2012;43:e487-8.

14. Lippi G, Montagnana M, Aloe R, Cervellin G. Highly sensitive troponin immunoassays: navigating between the Scylla and Charybdis. Adv Clin Chem 2012;58:1-29.

15. Apple FS, Morrow DA. Delta cardiac troponin values in clinical practice: are we ready to move absolutely forward to clinical routine? Clin Chem 2012;58:8-10.

16. Cardinaels EP, Mingels AM, Jacobs LH, et al. A comprehensive review of upper reference limits reported for (high-)sensitivity cardiac troponin assays: the challenges that lie ahead. Clin Chem Lab Med 2012;50:791-806.

17. Biomarkers definitions working group. Biomarkers and surrogate endpoints: preferred definitions and conceptual framework. Clin Pharmacol Ther 2001;69:89-95.

18. Thygesen K, Mair J, Giannitsis E, et al. The study group on biomarkers in cardiology of the ESC working group on acute cardiac care. How to use high-sensitivity cardiac troponins in acute cardiac care. Eur Heart J 2012;33:2252-7.

19. Christ M, Bertsch T, Popp S, et al. Highsensitivity troponin assays in the evaluation of patients with acute chest pain in the emergency department. Clin Chem Lab Med 2011;49:1955-63.

20. Lippi G, Montagnana M, Salvagno GL, Guidi GC. Potential value for new diagnostic markers in the early recognition of acute coronary syndromes. Can J Emerg Med Care 2006;8:27-31.

21. Lippi G. Biomarkers of myocardial ischemia in the emergency room: cardiospecific troponin and beyond. Eur $\mathrm{J}$ Intern Med 2013;24:97-9.

22. Lackner KJ. Laboratory diagnostics of myocardial infarction - troponins and beyond. Clin Chem Lab Med 2013;51:83-9.

23. Lippi G, Plebani M, Di Somma S, et al. Considerations for early acute myocardial infarction rule-out for emergency department chest pain patients: the case of copeptin. Clin Chem Lab Med 2012;50:243-53.

24. Di Somma S, Navarin S, Giordano S, et al. The emerging role of biomarkers and bioimpedance in evaluating hydration status in patients with acute heart failure. Clin Chem Lab Med 2012;50:2093-105.

25. Pope JH, Aufderheide TP, Ruthazer R, et al. Missed diagnosis of cardiac ischemia in the emergency department. New Engl J Med 2000;342:1163-70.

26. Larson DM, Menssen KM, Sharkey SW, et al. "False-positive" cardiac catheterization laboratory activation among patients with suspected ST-segment elevation myocardial infarction. JAMA-J Am Med Assoc 
2007;298:2754-60.

27. Hoekstra JW, 0'Neil BJ, Pride YB, et al. Acute detection of ST-elevation myocardial infarction missed on standard 12-led ECG with a novel 80-lead real-time digital body surface map: primary results from the multicenter OCCULT MI Trial. Ann Emerg Med 2009;54:779-88.

28. Peacock W, Hoekstra J, Krucoff M, et al. Early objective identification of chest pain patients at very low risk of 30 day adverse outcomes. Ann Emerg Med 2009;54:S2.

29. Parato VM, Mehta A, Delfino D, et al. Resting echocardiography for the early detection of acute coronary syndromes in chest pain unit patients. EchocardiographyJ Card 2010;27:597-602.

30. Lee B, Chang AM, Matsuura AC, et al. Comparison of cardiac risk scores in ED patients with potential acute coronary syn- drome. Crit Pathw Cardiol 2011;10:64-8.

31. Mehta SR, Granger CB, Boden WE, et al. TIMACS Investigators. Early versus delayed intervention in acute coronary syndromes. New Engl J Med 2009;360: 2165-75.

32. Katritsis DG. Optimal timing of coronary angiography and potential intervention in non ST-elevation acute coronary syndromes. Eur Heart J 2011;32:32-40. 